\title{
Results of Long-Term Follow-Up Observations of Blepharoptosis Correction Using the Palmaris Longus Tendon
}

\author{
SangKeun Park $\cdot$ YongHo Shin
}

Received: 21 January 2008/Accepted: 3 April 2008/Published online: 30 April 2008

(C) The Author(s) 2008

\begin{abstract}
Background The frontalis sling procedure is a useful approach for correcting severe blepharoptosis. However, blepharoptosis often recurs after corrective surgery using the tensor fascia lata. Good results without recurrence after a modified Fox method were obtained using the palmaris longus tendon. This study examined the safety and validity of the surgical method using the palmaris longus tendon through long-term follow-up observations.

Methods To reduce the rate of recurrence, the highest point on the pentagon of the Fox method was fixed to the frontalis fascia and frontalis muscle. It was fixed once again to the area $1 \mathrm{~cm}$ above the highest point. This approach remarkably reduces the incidence of recurrence by fixing the pentagon of the Fox method not only to the palmaris longus tendon but also to the frontalis fascia and again to the frontalis muscle. A modified Fox method using the palmaris longus tendon was used to treat 16 eyelids of 10 patients. A senior surgeon performed the procedure in all cases under local anesthesia.

Results The mean follow-up period was 51 months (range $=18-86$ months). There was no case of blepharoptosis recurrence and a good field of view was secured after surgery. Long-term follow-up revealed that the visual field had been well secured with a mean MRD1 of $3.1 \mathrm{~mm}$. The eyelids were well maintained without any postoperative adverse reaction such as exposure keratitis.

Conclusion The palmaris longus tendon as useful donor material does not lead to recurrence of blepharoptosis, which is often encountered when the tensor fascia lata is used. The modified Fox method using the palmaris longus
\end{abstract}

S. Park $(\square) \cdot$ Y. Shin

BK DongYang Plastic Surgery Clinic, Seoul, Republic of Korea

e-mail: pspsk@naver.com tendon can be an effective and valid surgical approach that produces both immediate and long-term results.

Keywords Blepharoptosis - Palmaris longus . Long-term follow-up · Modified Fox method

Blepharoptosis is classified into three levels: mild, moderate, and severe [1]. The treatment for severe cases of blepharoptosis is quite limited. The frontalis sling procedure, along with frontalis muscle transfer, is one of several excellent procedures for correction of severe blepharoptosis. The frontalis sling procedure is suitable for patients whose levator palpebralis muscle has almost no power or when a levator resection procedure had failed. The tensor fascia lata is used mainly for the frontalis sling procedure in severe blepharoptosis cases. Nevertheless, it is difficult to obtain the tensor fascia lata in children younger than 3 years of age because they are undeveloped and general anesthesia would be essential for surgery [2]. Therefore, Kurihara et al. [3] introduced a surgical procedure for blepharoptosis that uses the palmaris longus tendon which was used mainly in hand and facial reconstructions, and there have been a variety of reports on the procedure since. The palmaris longus tendon may be used in pediatric patients and can be collected easily within 10 min under local anesthesia. The method for incorporating the palmaris longus tendon is widely known.

In this study severe blepharoptosis cases were corrected using the autologous palmaris longus tendon without functional abnormalities. The method of Lam et al. [2] or Kurihara et al. [3] used a ligament divided into three ligaments. However, in this study the ligament was fixed in a pentagonal shape. 6-0 Nylon was used to fix the ligament to three sites on the palpabral plate. Two sites, one on the 
right and one on the left side, were fixed to a part of the frontalis muscle immediately below the eyebrow bilaterally. Two strips of the frontalis muscle were gathered together $1 \mathrm{~cm}$ above the eyebrow. Secondary fixation was performed twice and the dispersion of power was arranged precisely to be equal on each side (Fig. 1).

Corrective surgery using the palmaris longus tendon was performed on 16 palpebrae in 10 patients. The postoperative palpebrae were satisfactory. This article reports the long-term follow-up results.

\section{Materials and Methods}

Corrective surgery was performed on ten patients with severe blepharoptosis using the palmaris longus tendon from October 2000 through June 2007. The mean followup period was 51 months. There were 6 females and 4 males. Nine patients had congenital blepharoptosis and one had traumatic blepharoptosis. The range of the levator palpebral muscle was within $4 \mathrm{~mm}$ in all cases. The preoperative tests included MRD1 of blepharoptosis, uppereyelid function, superior rectus muscle function, tear secretion, Bell's phenomenon, and the Marcus-Gunn phenomenon. All procedures were performed using the palmaris longus tendon, which was harvested under local anesthesia. There was no case in which harvesting could not be performed because of nonexistence of the palmaris longus tendon. The presence and position of the palmaris longus tendon before surgery were verified by making a "V" shape using a "two finger sign" with the thumb and index finger.

The procedure was performed under local anesthesia. The edge of the palpebra was incised approximately 5$7 \mathrm{~mm}$ and the tarsal plate was separated after the orbicularis oculi muscle had been put aside. The Visor's fascia of the tarsal plate was separated and the pure tarsal plate was exposed. The modified Saeed and Ray method was used to harvest the palmaris longus tendon [4]. A $0.5-\mathrm{cm}$ incision was made on the wrist folds under local anesthesia and the palmaris longus tendon was exposed. Following down the exposed palmaris longus tendon, the tendon was separated in the direction of the tendon and the upper part of the tendon was verified. A $1-\mathrm{cm}$ incision was made $7-10 \mathrm{~cm}$ above the wrist folds. The palmaris longus tendon that was exposed from the distal incision site was separated into two strips using a No. 15 scalpel and extended into the proximal part. Two strips, one half and two thirds of the tendon, were used instead of the entire palmaris longus tendon. The mean width and length of the palmaris longus tendon was $4-5 \mathrm{~mm}$ and $7-10 \mathrm{~cm}$, respectively. These were cut and divided into two halves. The depth was shortened and the length was increased twofold before use. The harvested palmaris longus tendon was fixed firmly from the upper edge of the tarsal plate to the limbus line and to three sites in the pupillary line using 6-0 nylon. An approximately $2-\mathrm{cm}$ incision was made immediately above the eyebrow after firmly fixating the tarsal plate.

Each end of the tendon was pulled through the incision site through the soft tissues of the upper eyelid. This was done to verify and adjust the shape of the eyelid. The tendon was pulled with proper tension so that the edge of the upper eyelid was between the superior edge of the pupil and the limbus. The tendon was fixed to the subfollicular plane below the eyebrow. Each end of the tendon was then pulled together and fixed twice $1 \mathrm{~cm}$ above the eyebrow (Fig. 1). The skin of the eyelid was sutured using 7-0 silk and that of the upper eyebrow was sutured using 5-0 vicryl and 6-0 nylon. The suture material was removed one week later.

\section{Results}

Surgery was performed on ten patients using the palmaris longus tendon from October 2000 through June 2007. There were 4 males and 6 females with a mean age of 35 years. Of these subjects, one patient underwent surgery again 1 week after the initial surgery because of a slight slackening due to trauma in the left eye. The results were
Fig. 1 Schematic diagram of the procedure. The red color indicates the tendon. Note the suture placement on three points on the tarsal plate. The dotted rectangular circle indicates the dissection above the frontalis muscle

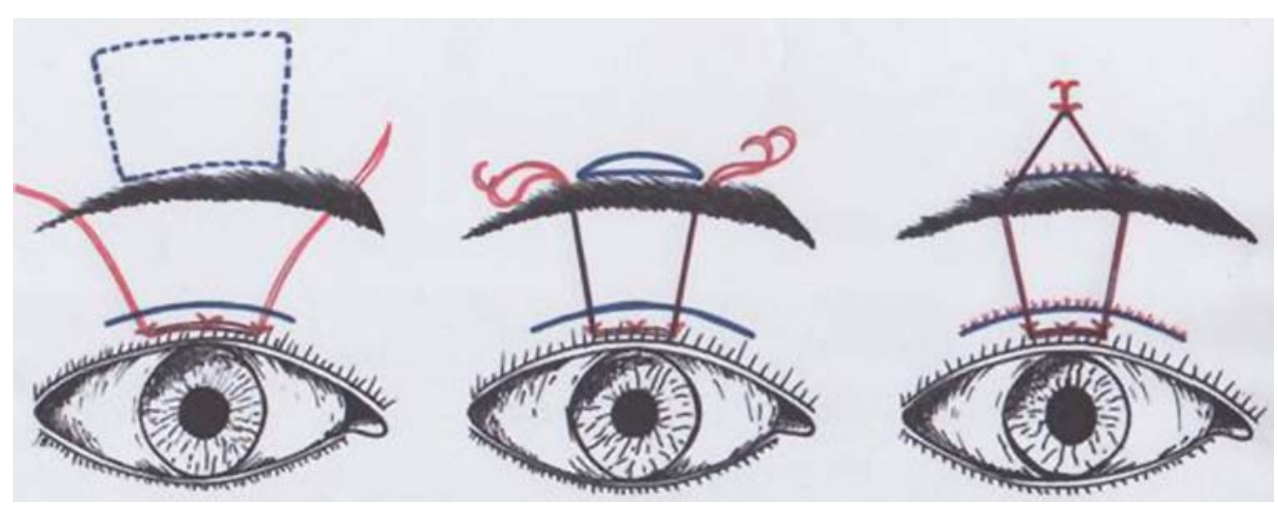


Table 1 The preoperative findings and postoperative results

\begin{tabular}{llllll}
\hline Patient, age & Gender & $\begin{array}{l}\text { Function of } \\
\text { levator palpebra } \\
\text { before surgery }(\mathrm{mm})\end{array}$ & $\begin{array}{l}\text { Postoperative } \\
\text { MRD1 }(\mathrm{mm})\end{array}$ & $\begin{array}{l}\text { Follow-up } \\
\text { observation } \\
\text { period } \\
(\mathrm{month})\end{array}$ & Complications \\
\hline 1,23 & Male & 0 & 3.0 & 56 & None \\
2,66 & Female & 4 & 3.5 & 34 & None \\
3,48 & Male & 3 & 3.0 & 77 & None \\
4,54 & Male & 4 & 3.5 & 39 & None \\
5,33 & Female & 2 & 3.0 & 46 & None \\
6,21 & Female & 0 & 2.5 & 86 & None \\
7,20 & Female & 0 & 3.0 & 64 & None \\
8,31 & Female & 4 & 3.5 & 71 & None \\
9,35 & Male & 2 & 3.0 & 18 & None \\
10,19 & Female & 2 & 3.0 & 20 & None \\
\hline
\end{tabular}

satisfactory and without complications. After surgery, the mean MRD1 value was $3.1 \mathrm{~mm}$ after follow-up of 51.1 months. The results secured good vision and aesthetic enhancement with no recurrence (Table 1; Figs. 1-4).

On the other hand, two of the ten cases treated using freeze-dried lyophilized tensor fascia lata recurred in 6 and 15 months. Therefore, corrective surgery using the palmaris longus tendon of the autogenous tissues provided more satisfactory results. All ten patients who underwent the procedure with the palmaris longus tendon had a satisfactory eyelid shape. Slight slackening of the eyelid was observed 3-6 months after surgery, but the level of slackening did not require additional correction. All patients
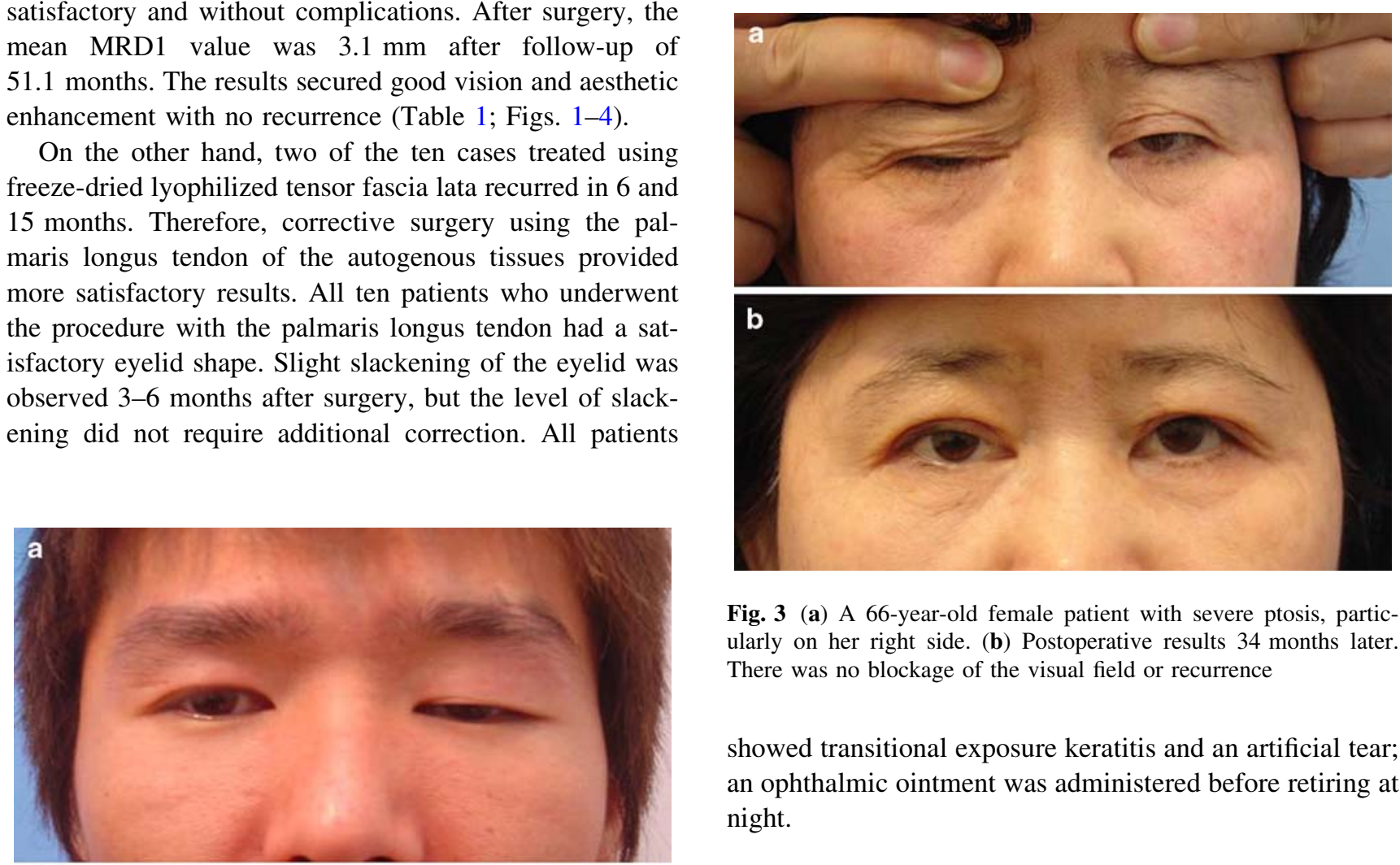

Fig. 3 (a) A 66-year-old female patient with severe ptosis, particularly on her right side. (b) Postoperative results 34 months later. There was no blockage of the visual field or recurrence

showed transitional exposure keratitis and an artificial tear; an ophthalmic ointment was administered before retiring at night.

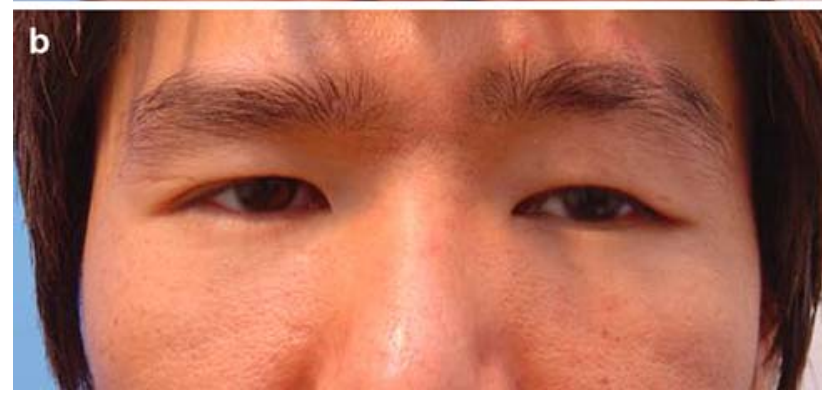

Fig. 2 (a) A 23-year-old male patient with severe ptosis on the left side. (b) Eighteen months after the frontalis sling procedure using the palmaris longus tendon

\section{Discussion}

The materials used for the frontalis sling procedure include artificial materials such as prolene, a silicone sling, supramid suture, braided polyester, expanded polytetrafluoroethylene (Gore-Tex), and autogenous tensor fascia lata and palmaris longus tendon. In addition, freeze-dried tensor fascia lata, which is a treated tensor fascia lata of humans, can also be used. Nevertheless, there are reports of poor long-term results in cases treated using the tensor fascia lata. Wilson and Johnson [5] went so far as to state 

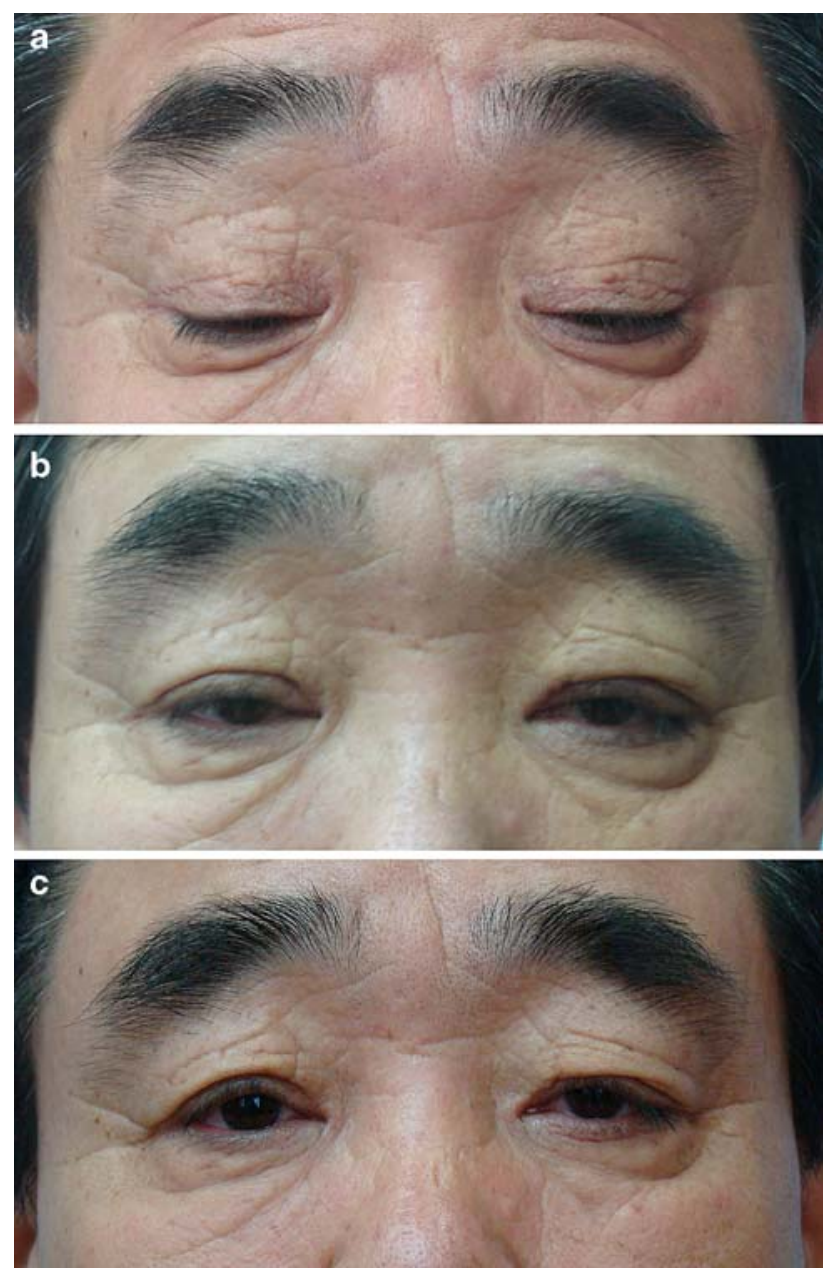

Fig. 4 A 54-year-old male patient with severe bilateral blepharoptosis. (a) The preoperative finding revealed that the levator function was zero. (b) Six months after the frontalis sling procedure using the lyophilized tensor fascia lata. (c) Thirty-nine months after the frontalis sling procedure using the palmaris longus tendon

that half the procedures in which the tensor fascia lata was used failed 8-9 years after surgery. However, there were significantly different results between the autogenous fascia lata and lyophilized fascia lata. Wassermann et al. [6] reported recurrence rates of $4.2 \%$ with the autogenous fascia lata and $51.4 \%$ with the lyophilized fascia lata. On the other hand, the palmaris longus tendon might be used in children under the age of 3 years and would be an autogenous tissue with less recurrence after surgery. Wong et al. [7] examined 14 patients and 15 eyelids and reported that there was no recurrence after surgery using the palmaris longus tendon during a mean follow-up period of 7.8 years. Therefore, the palmaris longus tendon, with its advantage of being an autogenous tissue and having long-term endurance after surgery, might be an ideal material for blepharoptosis correction compared with the autogenous tensor fascia lata.
The problem in using the palmaris longus tendon is that there are many anatomical variations. Reimann et al. [8] reported in a study of 1600 cases that approximately $21.8 \%$ of patients had anatomical abnormalities, the most common being an absence of the palmaris longus tendon (in up $12.8 \%$ of cases). Lee [9] reported the bilateral and unilateral existence of the palmaris longus tendon in $70 \%$ and $85 \%$, respectively. On the other hand, Won et. al. [10], in a study of 1000 patients, reported the bilateral absence of the palmaris longus tendon in 11 patients $(1.1 \%)$ and unilateral absence in $32(3.2 \%)$. A study in China reported that the incidence of bilateral and unilateral absence was $1.2 \%$ and $3.3 \%$, respectively [11]. This means that there are significant anatomical variations between Asians and Caucasians with respect to the palmaris longus tendon. Cases where the palmaris longus tendon is missing often have other anatomical abnormalities. A typical example would be the plantaris tendon [12]. However, the plantaris tendon has relatively no association with the absence of the flexor digitorum superficialis of the fifth finger [13]. Other than that, the variation of the superficial palmar arch or cases in which the muscle and tendon parts are switched and the muscle part exists distally might be the cause of carpal tunnel syndrome [14, 15]. In addition, it should be noted that there could be a multitude of anatomical variations that need to be considered before surgery. Reimann et al. [8] reported that more females lacked the palmaris longus tendon on the left side. However, recent studies showed that there were no significant differences in the presence of the palmaris longus tendon between genders or between the left and right sides [10-12]. The mean length of the palmaris longus tendon in Asians is $124 \mathrm{~mm}$ for males and $108 \mathrm{~mm}$ for females, and the mean widths are $45 \mathrm{~mm}$ for males and $40 \mathrm{~mm}$ for females [16]. There are many methods for verifying the introduction of the palmaris longus tendon, including the "two finger" sign, "finger fan out" sign, "closed fist" sign, "lotus" sign, and "flexion of the wrist against resistance." All these methods examine for presence of the palmaris longus tendon [17].

The disadvantage of the eyelid bulging out was minimized using a one-half to one-third portion of the palmaris longus tendon instead of the entire tendon. In particular, Asians do not like the baseline of the double-folded eyelid bulging out, and it is best to perform surgery using a minimum thickness palmaris longus tendon. A complete resection of the palmaris longus tendon does not lead to functional abnormalities such as a reduction in finger gripping and grabbing power. Nevertheless, only a part of the palmaris longus tendon was used to prevent wasting the tendon [18].

The methods for fixing the palmaris longus tendon differ between authors. First, Kurihara et al. [3] attempted to achieve symmetry by dividing one strip into three. 
Chung et al. later utilized the same method [19]. Typical fixation methods using the existing tensor fascia lata include the Fox method and the Crawford method. Later, many modified approaches were introduced, including one triangle (Fox variation), two triangles (Crawford variation), and pentagonal and rectangular forms. This study used a pentagonal form, which is a modified Fox method that allows a firmer fixation than the triangle form that uses one fixing point. The pentagonal form uses two fixation points and reduces the incidence of recurrence. With respect to suturing the palmaris longus tendon onto the eyelid, distortion of the eyelid shape was minimized by allowing five chances to adjust the eyelid: (1) when suturing the palmaris longus tendon onto the tarsal plate, $(2,3)$ when suturing it to the eyebrow area (left and right), (4) when tying up two strips of the palmaris longus tendon into one, and (5) when fixing it onto the frontalis muscle. Fixation in the eyebrow area would be carried out into the subfollicular layer. According to Hwang et al. [20] the reason could be that the layer of the frontalis muscle would extend to the inner area of the eyebrow, to the upper edge of the eyebrow centrally, and to the area $0.5 \mathrm{~cm}$ below the upper edge of the eyebrow laterally. Therefore, fixations of the layer of the frontalis muscle onto the eyebrow can have the effect of suturing onto the frontalis muscle itself. For target locations of the edge of the eyelid, an excess of $1 \mathrm{~mm}$ on one side compared with the opposite side was corrected in unilateral eyelid surgery while the level of lagophthalmos was considered for the area located 1-2 $\mathrm{mm}$ above the corneal limbus in bilateral eyelid surgery before undertaking such corrective surgery. This level was lower than the area $1 \mathrm{~mm}$ above the corneal limbus targeted by Lam et al. [2]. This means that postoperative complications such as exposure keratitis could last a long time if a surgeon positions the palmaris longus tendon into an area considered to be "good" in Manners et al. [21] postoperative assessment. Therefore, it is believed that the incidence of complications can be reduced and an adequate field of view and good aesthetic outcome can be provided by securing a sufficient field of view and positioning the fixation in an area $1 \mathrm{~mm}$ below the corneal limbus [21]. To reduce the probability of exposure keratitis, the patients were asked to administer an artificial tear solution or ointment before bedtime for a minimum of 6 months to 1 year.

A preoperative plan might be the most important index for determining the suitability of surgery. Achieving left and right symmetry during surgery is often very difficult because of local anesthesia or hematoma. In such cases, it would be best to confirm the difference between the left and right sides in a lid lag, and at the same time the surgeon should perform surgery based on the preoperative plan. Even in long-term follow-up observations after surgery, the position of the eyelid edge allows verification of the visual field and maintenance of the location that is not associated with an excessive or underestimated correction. This article reports this method based on the idea that the postoperative MRD1 is $3.1 \mathrm{~mm}$ and a correction of severe blepharoptosis using the palmaris longus tendon can provide excellent long-term results, bilateral symmetry, and less recurrence.

\section{Conclusion}

In this study the frontalis sling procedure using the palmaris longus tendon was performed to correct blepharoptosis of 16 eyelids in 10 patients from October 2000 through June 2007. The outcome was examined using longterm follow-up observations of these eyelids. With respect to the technique, the tendon was fixed in a pentagonal shape. The tendon was fixed to the tarsal plate at three sites using 6-0 nylon. The tendon was also fixed superiorly to the part of the frontalis muscle immediately below the eyebrow at two sites, one on the right and one on the left. The two strands of the frontalis muscle $1 \mathrm{~cm}$ above the eyebrow were tied together so that the second fixation was performed twice to prevent recurrence. The power dispersion was arranged precisely to be even on the right and the left sides. Satisfactory results were obtained immediately after surgery and after a long-term follow-up period. Overall, the modified Fox method using the palmaris longus tendon is quite a useful approach for correcting severe blepharoptosis and can provide good immediate and long-term results.

Open Access This article is distributed under the terms of the Creative Commons Attribution Noncommercial License which permits any noncommercial use, distribution, and reproduction in any medium, provided the original author(s) and source are credited.

\section{References}

1. Beard C (1998) Examination and evaluation of the ptosis patient. In: Smith's Ophthalmic Plastic and Reconstructive Surgery, 2nd edn. C.V. Mosby, St. Louis, 339 pp

2. Lam DSC, Lam TP, Chen IN, Tsang GH, Grandhi SR (1996) Palmaris tendon as a new autogenous material for frontalis suspension surgery in adults. Eye 10:38-42

3. Kurihara K, Kojima T, Marumo E (1984) Frontalis suspension for blepharoptosis using palmaris longus tendon. Ann Plast Surg $13: 274$

4. Saeed WR, Kay SP (1993) Harvest of palmaris longus tendon technique. J Hand Surg 18:583-584

5. Wilson MB, Johnson RW (1991) Congenital ptosis: long-term results of treatment using lyophilized fascia lata for frontalis suspension. Ophthalmology 98:1234-1237

6. Wasserman BN, Sprunger DT, Helveston EM (2001) Comparison of materials used in frontalis suspension. Arch Ophthalmol 119:687-691

7. Wong CY, Fan DSP, Ng JSK, Goh TYH, Lam DSC (2005) Longterm results of autogenous palmaris longus frontalis sling in children with congenital ptosis. Eye 19:546-548 
8. Reimann AF, Daseler EH, Anson BJ, Beaton LE (1944) The palmaris longus muscle and tendon; a study of 1600 extremities. Anat Rec 3:275-278

9. Lee M (1987) Tendon injuries. In: Crenshaw AH (ed) Campbell's operative orthopedics, vol 1. C.V. Mosby, St Louis, p 175

10. Won CH, Chang BS, Chang MS (1994) Palmaris longus in Korean. J Kor Orthop Assoc 29(6):1561-1563

11. Sebastin SJ, Puhaindran ME, Lim AYT, Lim IJ, Bee WH (2005) The prevalence of absence of the palmaris longus-a study in a Chinese population and a review of the literature. J Hand Surg [Br] 30(5):525-527

12. Vanderhooft E (1996) The frequency and relationship between the palmaris longus and plantaris tendon. Am J Oprthop 25:38-41

13. Sebastin SJ, Lim AYT, Wong HB (2006) Clinical assessment of absence of the palmaris longus and its association with other anatomical anomalies-a Chinese population study. Ann Acad Med Singapore 35:249-253

14. O'Sullivan E, Mitchell BS (2003) Association of the absence of palmaris longus tendon with an anomalous superficial palmar arch in the human hand. J Anat 201:405-408
15. Natsis K, Levva S, Totlis T, Anastasopoulos N, Paraskevas G (2007) Three-headed reversed palmaris longus muscle and its clinical significance. Ann Anat 189:97-101

16. Ito MM, Aiko M, Kida MY, Ishii S, Kumaki K, Tanaka S (2001) Length and width of the tendinous portion of the palmaris longus: a cadaveric study of adult Japanase. J Hand Surg (Am) 26:706710

17. Bhattacharya V, Reddy GR (2005) The palmaris longus tendonrationality of the finger maneuvers. Br J Plast Surg 58:419-421

18. Sebastin SJ, Lim AYT, Bee WH, Wong TCM, Methil BV (2005) Does the absence of the palmaris longus affect grip and pinch strength? J Hand Surg [Br] 30(4):406-408

19. Chung KC, Ma HS, Lee C (1986) Correction of congenital blepharoptosis using palmaris longus tendon. J Kor Soc Plast Reconstr Surgeons 13(2):405-410

20. Hwang K, Kim DJ, Hwang SH (2005) Insertion of frontalis muscle to blepharoptosis repair. J Craniofac Surg 16(6):965-967

21. Manners RM, Tyers AG, Morris RJ (1994) The use of prolene as a temporary suspension material for brow suspension in young children. Eye 8:346-348 